\title{
Study method for pedestrian behaviour in the area of pedestrian crossings located at tram stops
}

\author{
Marek Bauer ${ }^{1, *}$, and Wiesław Dźwigoń ${ }^{1}$ \\ ${ }^{1}$ Cracow University of Technology, Faculty of Civil Engineering, Cracow, Poland
}

\begin{abstract}
The paper presents the problem of pedestrian safety at stops, with stops designed for opposite directions. Stop areas with a predominantly pedestrian traffic, where pedestrians cross tram tracks, are not protected by traffic signals. The article presents a method for conducting a study to identify types of pedestrian and tram driver behaviour, developed on the basis of a pilot study. This method helps to evaluate, inter alia, the tendency of pedestrians to risk going across the tram track before an oncoming tram, depending on how far it is from the pedestrian crossing. It also helps to determine the probability of the tram slowing down or emergency braking when crossing the pedestrian crossing, and the probability of difficulties with the departure of the tram, caused by pedestrians.
\end{abstract}

\section{Introduction}

Nowadays, trends can be seen in European cities aiming to increase the role of walking and public transport [1]. This is especially true of downtown areas, where excessive car traffic degrades urban spaces and deteriorates living conditions of the citizens [2]. The main reason for this is the considerable and often increasing number of journeys within the downtown area which cannot be satisfied with car transport. The seemingly favourable development of new roads causes even more congestion and takes away space from pedestrians who should be given special priority in the city centre [3]. Therefore, the gradual shift from the previous policy enabling a free use of the car in city centres is a highly desirable phenomenon, especially for medium-sized and large cities [4]. The scope of possible actions is relatively broad and includes solutions facilitating public transport traffic, supporting walking and cycling and, an area which still remains unaccepted by some users of the transportation system, restricting the car [5]. Only if used skilfully, can these solutions create a chance for success and keep the area attractive for the public and visitors while ensuring good access to the area. The simultaneous use of solutions favouring vulnerable road users and public transport vehicles is only seemingly an easy task. Above all, pedestrians expect unrestrained access. Passengers of public transport expect a quick connection to the downtown area which must be attractive, i.e. relatively quick, particularly when it comes to the tram network which is more susceptible to traffic disturbances [6]. Ensuring the right balance between the degree of enhancement of pedestrian traffic and public transport vehicles has considerable importance. The most important problems observed in city centres are conflicts between pedestrians and trams within tram stops, particularly when the stops are located near busy

*Corresponding author: mbauer@pk.edu.pl 
footways with pedestrian streams crossing stops when the trams are arriving even if they do not want to use the tram. The key role in this sense is played by the diversity of pedestrian behaviours and variable awareness of the traffic and braking capabilities of the trams.

The present study discusses the issues related to the comfort and safety of using transport infrastructure in the potential conflict zone between pedestrian and tram traffic. The original proposal for the study methodology includes road user behaviours (pedestrians and tram drivers) in the vicinity of pedestrian crossings located near tram stops. Conflicting interests of vulnerable road users (safety and comfort) and passengers of public transport (comfort and speed) must be reconciled in these areas.

\section{Current approach to the study of conflicting situations between pedestrians and trams}

The number of accidents and collisions that took place on individual tram stops is usually not relevant, as the accidents take place relatively rarely [7]. Moreover, studies of hazardous situations enable a description of atypical or temporary solutions - which should be tested prior their eventual dissemination. On the basis of the definition accepted in 1997 in Oslo, it can be stated that "pre-conflicting road user behaviour is a situation, when the users violate the established thresholds for road behaviour, yet which do not result in a road accident, and which do not force a change in the behaviour of other road users in order to avoid the accident" and "traffic conflict is a situation, when at least one of the users takes action to prevent an accident". The basic indexes evaluating traffic conflicts include:

- CS (Conflicting Speed) - this is the speed of road user, who began evasive actions (just before starting the action) [8];

- TA (Time to Accident) - time which passes from the taking of an evasive action by at least one road user to the moment of a potential accident, if both road users continued movement at the same speed and the same direction;

- TTC (Time To Collision) - time to the collision of two objects, if their trajectories and speeds remain unaltered. The lower value of TTC means a higher likelihood of the accident with more serious consequences [9].

However, the above indexes are more appropriate for the study of "vehicle-vehicle" conflicts than for "vehicle-pedestrian" conflicts [10], as they concern independent vehicles already in motion. Whereas in the case of "vehicle - pedestrian" conflict, particularly within stops, the conflicting situations often stem from the incorrect evaluation of the possibility to enter the track (pedestrian) or incorrect evaluation of the possibility to leave the stop (tram driver).

Therefore, techniques for the study of road user behaviour have been introduced and enhanced. They are typically conducted in a traditional form, with observers detecting conflicting situations and entering the types and numbers to the questionnaires, often as supplementary research to pedestrian and vehicle traffic measurements. Currently, a video technique is increasingly commonly used for this purpose enabling a lower number of participants to the observations and offering better accuracy of the conducted study. However, independently of the technical feasibilities of research, selection of the observed behaviours and correct, unambiguous definition of conflicting situations remain the most important issue. It largely depends on the approach of the researchers. A major step in the development of traffic conflicts was the research conducted within the project CiViTAS CARAVEL [11], implemented in order to enhance the attractiveness, comfort and safety of city transport. In the study, conflict measurements included three types of stops: with passenger exchange in the lane, with elevated lane (so called Vienna-like tram stop) and with elevated lane and island platform. The study did not analyse opposite stops located in areas with shared tram-pedestrian function. 


\section{Proposed method for the study of conflict situations between pedestrians and trams within the area of opposite tram stops}

Conflicting situations between pedestrians and trams within tram stops typically take place while pedestrians are crossing tracks - and the proposed study will be most relevant in this field. Moreover, only crossings without traffic lights were included. In the proposed method, the level of safety is assessed using the analysis of frequency and potential outcomes of hazardous situations (traffic conflicts). For obvious reasons, the method must comply with the current legislation including the rules on priority. These rules are discussed in Article 13 of the Road Traffic Act [12]; they demonstrate the obligation to cross the track separated from the traffic lane only in the designated area, and where a passenger island situated on a public transport stop is connected to a pedestrian crossing, reaching and leaving the stop is allowed solely on the crossing. However, the most important issue is that the pedestrian is obliged to take special care prior to entering the traffic lane. While on the crossing, the pedestrian has priority over all vehicles, including trams. However, the pedestrian does not have priority over a tram while waiting to enter the pedestrian crossing, which is regulated by Article 14 of the above Act, prohibiting (among others) stepping onto the lane directly before an approaching vehicle, including pedestrian crossings. An issue to be addressed is evaluating the safe distance from the tram while entering the pedestrian crossing. The pedestrian is not allowed to step onto the tracks before an approaching tram, whereas the tram driver approaching a pedestrian crossing must maintain special attention and travel at a speed enabling him to stop before the crossing if there is a pedestrian on the crossing.

The proposed method includes the general behaviour of pedestrians who cross tracks as well as the behaviour of tram drivers. In principle, the method is to be used for determining the use of infrastructure dedicated to pedestrian traffic, as well as for determining the frequency of traffic conflicts to decrease their prevalence. It is also intended to help with determining the causes of hazardous behaviour of pedestrians and tram drivers. In the proposed method, pedestrian traffic volumes are recorded on the entire width of pedestrian crossings and outside them. Pedestrian traffic volumes are recorded for both possible traffic directions and four possible tram positions: empty tram tracks - no approaching trams (on both sides of pedestrian crossings), tram arriving at a stop (stopping before the platform), tram leaving a stop after the passenger exchange is concluded and simultaneously: tram approaching the stop and tram leaving the stop ( opposite direction).

If crossing tram tracks is physically possible on both sides of the tram stop complex, traffic volumes must be recorded for both sides, even in the case when no dedicated pedestrian crossing is designated to one of the sides. The information on the percentage of people crossing outside the pedestrian crossing is part of a study of irregular events. When tracks are crossed at the approach of tramways nearing the stop, certain pre-conflicting and conflicting situations occur, therefore distances from the potential point of conflict were included. This is important because the results help to identify the tendencies of pedestrians to take the risk of crossing tracks when the tramway is nearing the potential conflict point. The following types of behaviours of pedestrians crossing tracks at the approach of a tram were assumed:

- a pedestrian crosses the tracks when there is no tram or the tram is at least 20 meters away, which means that it can stop safely and comfortably (for the passengers) travelling at a speed not exceeding $30 \mathrm{~km} / \mathrm{h}$ - in practice, this means a correct and safe crossing before an approaching tram (Figure 1); 


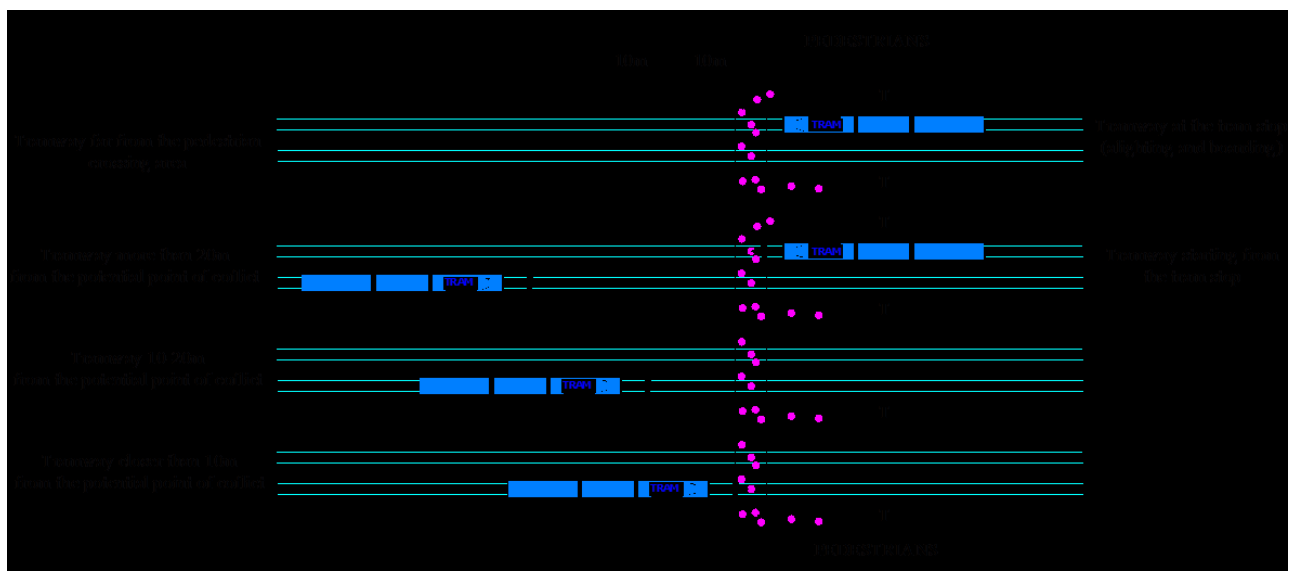

Fig. 1. Diagram of traffic conflicts between pedestrians and trams.

- a pedestrian crosses the tracks when the tram is at a distance of 10-20m, which corresponds to the possibility for a safe stop of the tram nearing the platform at the speed of up to approx. $16 \mathrm{~km} / \mathrm{h}$;

- a pedestrian steps on the tracks when the tram is at a distance lower than $10 \mathrm{~m}$, which is linked to a considerable risk of a pedestrian being hit, even at a relatively low speed;

- running onto the track when the tram is at a distance lower than $10 \mathrm{~m}$, which may result in the tram driver not taking any action (surprised by the appearance of the pedestrian).

On the other hand, in the case of recording pedestrian traffic volumes on a pedestrian crossing situated at the front of the stop with ongoing tram-passenger exchange, we have proposed the inclusion of three types of pedestrian behaviours:

- pedestrian entering the track when the tram remains at the stop;

- entering the track when the tram drives off from the stop - which results in a sound signal, possible deceleration at the start or even emergency stop of the tram;

- running onto the track when the tram accelerates from the stop, which is particularly dangerous due to the possibility of remaining unnoticed by the tram driver.

The following behaviour types were taken into consideration for tram drivers:

- free tram passage - no pedestrians;

- unproblematic start from the stop across the pedestrian crossing, due to the absence of pedestrians on and at the entrance to the crossing;

- passage across the crossing when pedestrians are waiting to step onto the crossing, but they are not on it - treated as correct passage across the crossing, often accompanied by sound signalling, tram deceleration or stop occurring sporadically;

- passage across the crossing when pedestrians are on the crossing, which is almost always accompanied by speed reduction or emergency stop;

- accelerating from the tram stop when pedestrians are on the crossing - depending on the situation, linked to a sound signal, deceleration or emergency stop.

The recorded behaviours of pedestrians and tram drivers must be accompanied by assessment indicators. A common approach is proposed, based on stratum weights. Determination of the share of individual behaviours described above and their reference to pedestrian and tram traffic volumes is possible. 


\section{Example of the method in practice: study of conflicting situations within the tram stop "Dworzec Główny Zachód" in Krakow}

The study of conflicting situations was performed following the presented methodology in several stop areas in Krakow. Figure 2 shows an example of the shares of passengers crossing the track in one of the analysed pedestrian crossings in relation to approaching trams.

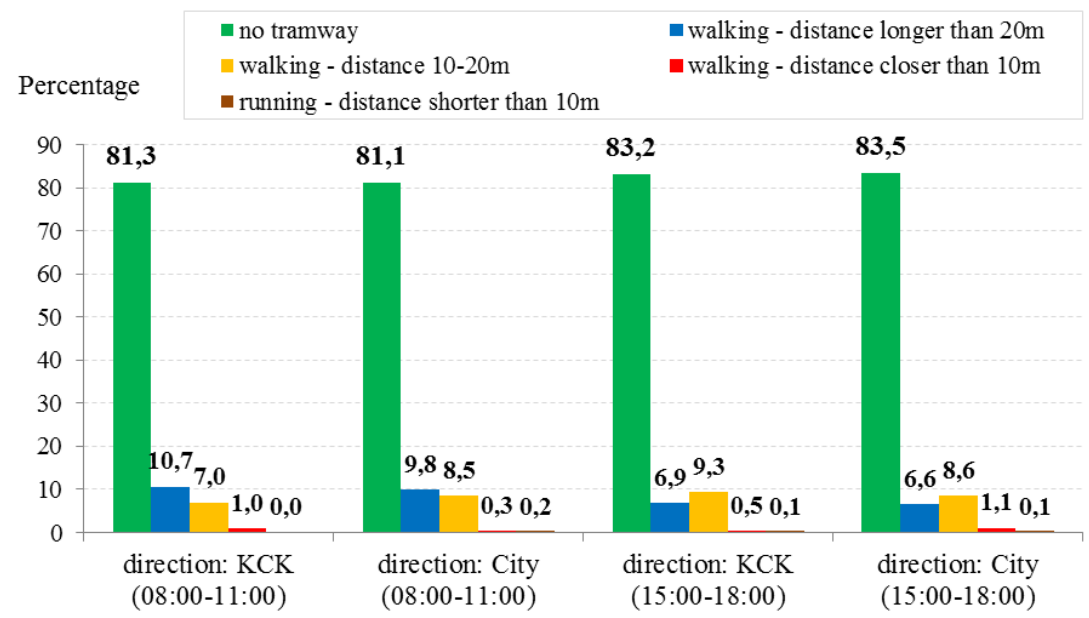

Fig. 2. Share of individual pedestrian behaviours on the analysed pedestrian crossing.

As can be seen, about $1 \%$ of pedestrians behave very riskily, in addition, to nearly $8 \%$ trying to pass when the tram is $10-20 \mathrm{~m}$ from the pedestrian crossing, which can also cause conflicts. And this "distance dilemma" will be particularly closely studied in the future. In this case, the tram track is located in the pedestrian area. Despite being separated, pedestrian and cycling traffic does occur there sporadically. It should be noted, however, that in reality almost the entire space between the above pedestrian crossings does not constitute a spatial barrier - and it is used by pedestrians to cross the tracks. It can also be noted that the share of passages outside of designated places is significant, amounting to $20 \%$ and that it varies depending on the direction and time of day. The drivers respond by using sound signals (7$16 \%)$, decelerating significantly $(4-11 \%)$ or even emergency stopping $(0-4 \%)$. This means that the drivers are prepared for unexpected behaviour of pedestrians and drive at safe speeds.

\section{Conclusions}

Measurement of hazardous situations occurring at stops and crossings requires further discussion. At this stage of analysis, other features were not investigated such as weather conditions, pedestrian age, visibility and parameters of pedestrian crossings. The absence of such wider studies means that the presented methodology and results should be considered as a pilot. It is important to define the safety and hazardous situations. Pedestrian behaviour is influenced by the location of sources and destinations of traffic and footways. The presented method can be - with some adaptation - used for the study of conflicting situations between trams and bicycles. 


\section{References}

1. P. Nijkamp, A.H. Perrel, Sustainable cities in Europe (Routledge, 2014)

2. K. Nosal, W. Starowicz, Evaluation of influence of mobility management instruments implemented in separated areas of the city on the changes in modal split, Arch. Transp. 35 pp. 41-52 (2015). doi:10.5604/08669546.1185186

3. K. Jamroz, W. Kustra, M. Budzyński, J. Żukowska, Pedestrian Protection, Speed Enforcement and Road Network Structure the key Action for Implementing Poland's Vision Zero, Transp. Res. Procedia. 14 pp. 3905-3914 (2016). doi:10.1016/j.trpro.2016.05.479

4. D.S. Vale, Transit-oriented development, integration of land use and transport, and pedestrian accessibility: Combining node-place model with pedestrian shed ratio to evaluate and classify station areas in Lisbon, J. Transp. Geogr. 45 pp. 70-80 (2015). doi:10.1016/j.jtrangeo.2015.04.009

5. A. Spickermann, V. Grienitz, H.A. von der Gracht, Heading towards a multimodal city of the future?, Technol. Forecast. Soc. Change. 89 pp. 201-221 (2014). doi:10.1016/j.techfore.2013.08.036

6. D. Esztergár-Kiss, C. Csiszár, Evaluation of Multimodal Journey Planners and Definition of Service Levels, Int. J. Intell. Transp. Syst. Res. 13 pp. 154-165 (2015). doi:10.1007/s13177-014-0093-0

7. L. Michalski, K. Jamroz, Technika konfliktów ruchowych i możliwości jej zastosowania, Transp. Miej. 8 pp. 149-152 (1989)

8. C. Hydén, The development of a method for traffic safety evaluation: the Swedish traffic conflict technique, Doctoral Thesis, Lund University, 1987

9. J.H. Kraay, A. van der Horst, Trautenfels - study: a diagnosis of road safety using the Dutch Conflict observation technique, DOCTOR (Report R-85-53) (Institute for Road Safety Research SWOV, Leidschendam, 1985)

10. S. Gaca, M. Kieć, A. Zielinkiewicz, Analyses of headways for assessment of risk rearend collision, in: 3rd Int. Conf. Road Saf. Simul., (Indianapolis, 2011)

11. A. Rudnicki, Innovations for sustainable urban transport: experiences from accomplishment of EU Civitas Caravel project (Cracow University of Technology, 2010)

12. Act of 20 June 1997 - Road Traffic Act, OJ 1997 No. 98 Pos. 602 (Poland, 1997) 